OAI-PMH: http://www.indteca.com/ojs/index.php/Revista Scientific/oai

Ensayo Original / Original Essay

\title{
Recapitulando la historia de la ciencia: de la atomización a la complejización
}

Autor: Héctor de León Bedoya Leguizamón Universidad de los Llanos, UNILLANOS hbedoya@unillanos.edu.co Villavicencio, Colombia https://orcid.org/0000-0002-3631-1973

Resumen

El problema ancestral de las divisiones disciplinares en el que algunas ciencias han venido siendo erigidas como superiores en detrimento de otras, aún continúa haciendo presencia en distintos ámbitos y niveles académicos e investigativos, creando bandos, relaciones de poder y subordinaciones. Pero, ¿cuándo y cómo empezó a fragmentarse la ciencia? ¿Cuál es la naturaleza de estas diferencias y aislamientos? ¿En qué medida el desconocimiento de las inflexiones, sinuosidades y mitos que subyacen la historia tradicional de la ciencia han contribuido a estos distanciamientos y desmembramientos disciplinares? ¿Qué aporte puede hacer el pensamiento complejo frente a estas disyuntivas en un futuro próximo? Razones por las cuales se ha hecho preciso trazarse el objetivo de remontarse en la noche de los tiempos para identificar algunos prejuicios y estereotipos históricos que han alcanzado a las comunidades académicas y científicas presentes, dificultándoles la gestión e interrelación del conocimiento, así como la consideración de lógicas de pensamiento distintas a las clásicas.

Palabras clave: historia de la ciencia; ciencia y sociedad; estructura del conocimiento.

Cómo citar este ensayo:

Bedoya, H. (2019). Recapitulando la historia de la ciencia: de la atomización a la complejización. Revista Scientific, 4(14), 390-410, e-ISSN: 2542-2987. Recuperado de: https://doi.org/10.29394/Scientific.issn.2542-2987.2019.4.14.19.390-410

Fecha de Recepción: 11-07-2019
Fecha de Aceptación:

28-09-2019
Fecha de Publicación:

05-11-2019 
Recapping the history of science: from atomization to complexization

\begin{abstract}
The ancestral problem of the disciplinary divisions in which some sciences have been erected as superiors to the detriment of others, still continues to be present in different fields and academic and research levels, creating sides, power relations and subordinations. But when and how did science start to fragment? What is the nature of these differences and insulations? To what extent the ignorance of the inflections, sinuosities and myths that underlie the traditional history of science have contributed to these distancing and disciplinary dismemberment? What contribution can complex thinking make in the face of these dilemmas in the near future? Reasons why it has become necessary to trace the objective of going back in the night of time to identify some historical prejudices and stereotypes that have reached the academic and scientific communities present, making it difficult for them to manage and interrelate knowledge, as well as the consideration of logical thinking different from the classic.
\end{abstract}

Keywords: history of science; science and society; structure of knowledge.

Date Received:

11-07-2019
Date Acceptance:

28-09-2019
Date Publication:

05-11-2019 


\section{Introducción}

Las divisiones que aún persisten entre las ciencias son cada vez más frecuentes, distantes e irreconciliables. Se trata de un fenómeno disgregativo de vieja data que ha obstaculizado las asociaciones disciplinales hasta llevar a sus pensadores a padecer toda serie de prejuicios bipolarizadores que restringen el avance de la ciencia. Por eso es habitual encontrar a muchos intelectuales y científicos preocupados por saber qué ha venido propiciando estas segregaciones, antagonismos, discriminaciones y escisiones en los ámbitos académicos e investigativos. Al respecto, Cavada (2004): analizó con preocupación que la ciencia, a medida que ha tratado de extender su alcance desde los fenómenos naturales a los problemas humanos y sociales, ha ido perdiendo su unidad epistemológica, es decir, el carácter universal de abordar y de concebir toda la variedad de fenómenos que pueden ser objetos de estudio; de ahí que se hable más bien de "las ciencias" que de la "ciencia", ya que subsiste una división que parece irreconciliable; teniendo así, de un lado las ciencias naturales que estudian lo material y del otro las ciencias sociales que estudian el comportamiento humano y social (pág. 3).

Empero, ¿cuál es la naturaleza de las oposiciones entre los partidarios de paradigmas científicos como las ciencias exactas, las naturales y las humanas y sociales? ¿A qué se deberá la marcada profundidad de estos deslindes? ¿Qué impide que se asocien? ¿Cuándo y cómo empezó a segmentarse la ciencia? ¿Acaso las radicales divisiones disciplinales son condición sine qua non para el progreso de la ciencia? Inquietudes que alimentaron en estas líneas el propósito de identificar algunos de los prejuicios y estereotipos históricos que han ralentizado el desarrollo de las dinámicas académicas y científicas, haciendo que la gestión e interrelación del conocimiento, así como la consideración de lógicas de pensamiento distintas a las clásicas, sea más tensa, dificultosa y lejana. 


\section{Desanudando algunas memorias de la ciencia}

Aunque se pueden reconocer varios factores influyentes en dichos fraccionamientos (de orden cultural, biológico, lingüístico, político, económico, epistemológico, psicológico, entre otros), hay un elemento que puede inicialmente arrojar luces fundamentales sobre este asunto, y es la historia de la ciencia misma. Pero antes de abrir algunas ventanas temporales es preciso agregar desde Alonso (2004): que, si bien "no hay un acuerdo general lo suficientemente consolidado sobre qué es la ciencia" (pág. 32); es importante partir de alguna consideración elemental sobre lo que es ella. Al respecto, la Real Academia Española (2014): la definió como un "conjunto de conocimientos obtenidos mediante la observación y el razonamiento, sistemáticamente estructurados y de los que se deducen principios y leyes generales con capacidad predictiva y comprobables experimentalmente" (pág. 549).

Significado básico en el que se pueden reconocer algunas etapas clásicas del método científico (observación, experimentación, conclusión, etc.) que se han venido presentado de manera paulatina y configuradora en la historia de la ciencia, yendo del saber crédulo y corriente al conocimiento escéptico y científico. Y aunque, como lo señaló García (2008a): el saber cotidiano es el modo común, corriente y espontáneo de conocer que se adquiere en el trato directo y cotidiano con los hombres y con las cosas, esto no significa que esté claramente diferenciado del saber culto, por el contrario tiene numerosas interrelaciones con el saber científico (pág. 203); de hecho, como lo señaló Bunge (1983), citado por García (2008b), la ciencia crece a partir del conocimiento común y le rebasa con su crecimiento (pág. 20).

En este prudente orden de ideas se puede reconocer que la historia de las ciencias ha sido un proceso de discernimiento lento, complejo y prolongado que ha dado lugar a muchas sentencias que han modelado su cuerpo doctrinal. $Y$ entre ellas hay una afirmación que ha estado en boga durante 
mucho tiempo en su "historia oficial" y a partir de la cual se dará inicio a algunos cuestionamientos, y es aquella tendencia común de remontarse tajantemente al siglo XVII para referirse al nacimiento de la ciencia y sus disciplinas. Verbigracia: Delgado (2011): indicó que "la ciencia emergió de la modernidad en el siglo XVII" (pág. 4); y Hernández (2014): afirmó que "la figura del especialista y las especializaciones científicas son un producto de la modernidad" (pág. 170); entre otros. No obstante, es preciso apuntar que estas declaraciones tradicionales no solo son históricamente deterministas sino que incurren en la consabida cronología lineal, gradual, acumulativa, reductora y continuativa que siempre se ha asumido, y en esto la historiografía científica también ha caído, la cual procede según Kuhn (2004a): "como un proceso comparado a la adición de ladrillos a un edificio, [donde] los científicos han ido añadiendo uno por uno hechos, conceptos, leyes y teorías al caudal de información que proporciona el libro de texto científico contemporáneo" (pág. 219).

En este mismo sentido es válido considerar que el proceso disyuntor que han sufrido las ciencias puede remontarse hasta la Antigüedad, es decir, desde aquellas difusas fronteras en las que el conocimiento se perdía entre la cotidianidad y la experimentación metódica, el mito y el logos, la irracionalidad y la racionalidad, lo sensible y lo abstracto, el sentido común y los hechos, en medio de cambios, permanencias, disociaciones, rupturas y alianzas; todos ellos fenómenos propios de la naturaleza polidimensional, multivariable y multifocalizada de todo proceso histórico; hasta consolidarse progresivamente en una manera de razonar que fue cada vez menos impresionable y más rigurosa que terminó dando a luz a la filosofía. Alumbramiento que suele situarse con gran unanimidad en el Siglo VI a.E.C. en Jonia, Grecia. ¿Grecia? Esto no deja de ser también una adjudicación simplista, helenocentrista y equivocada, puesto que Jonia está situada en la costa egea de la península de Anatolia (en Asia Menor, No en Europa), es decir, atiéndase bien a Dussel 
(2006): "apareció el pensamiento presocrático en la actual Turquía o en el sur de Italia y no en Grecia" (pág. 15); en otras palabras, eminentes filósofos de la antigua Grecia como Tales, Anaximandro, Anaxímenes, Heráclito, Jenófanes y Pitágoras fueron emigrados jonios; y añádase a esta refutación que, en esta transición in illo tempore, entre lo inverosímil y lo real, lo intuitivo y lo experimental, hubo otros pueblos que desde mucho antes ya venían haciendo adelantos científicos.

Basta con repasar, la historia de los egipcios y los caldeos para advertir el notable impulso que le concedieron a la ciencia occidental transmitiéndoles su patrimonio intelectual en medio de especulaciones extravagantes y convicciones esotéricas; de hecho son los registros científicos más antiguos de los que se tiene hoy noticia, hallados en papiros, jeroglíficos y escritos cuneiformes; y es que ya los egipcios hacían experimentos químicos, cálculos matemáticos y geométricos: fueron los primeros en conocer la duración del año solar, medir el área del rectángulo, resolver ecuaciones, hacer farmacopea, procedimientos quirúrgicos, medicina primitiva, construcción de arquitecturas monumentales, etc.; y los caldeos se entregaron a los estudios astronómicos: predijeron cambios meteorológicos y eclipses haciendo el calendario lunar, ideándose la medición angular y el sistema especial de $360^{\circ}$ con fracciones sexagesimales, etc.

Por tanto, muchas de aquellas prácticas pueden calificarse de científicas a la luz de la definición de ciencia que se citó al principio, puesto que en ellas hay claramente componentes esenciales del método científico clásico, por ejemplo, Schneider (2005a): reportó que "para Bertrand Russell, la afirmación [hecha por Anaximandro] de que todo estaba hecho de agua se debe considerar una hipótesis científica, ya que se podía demostrar empíricamente con la observación" (pág. 11); y páginas adelante, Schneider (2005b): recupera una admisión de Popper, señalando que "se puede describir la teoría de Parménides diciendo de ella que es la primera teoría hipotética- 
deductiva del mundo" (pág. 17). E incluso se puede rescatar que muchos de ellos fueron adelantados para su época: Tales de Mileto demostró teoremas geométricos que Euclides codificó tres siglos después; Anaximandro, Demócrito y Empédocles ya tocaban en sus discursos (dos mil años antes que Darwin) algunos conceptos adaptativos relacionados con la selección natural de especies; y Russell (2013a), observó que: "la teoría de los atomistas, estaba más próxima a la ciencia moderna que cualquier otra de la antigüedad" (pág. 20); Pitágoras por su parte dedujo que el planeta Tierra era una esfera (antes que Magallanes y Elcano lo aseveraran entre los años 1519 y 1521); Aristarco afirmó (1800 años antes que Copérnico) que el centro del sistema planetario es el Sol y no la Tierra, y sospechó (antes que Giordano Bruno) que las estrellas eran soles lejanos; entre otros adelantos.

No obstante, es justo agregar que la filosofía, sus escuelas de pensamiento y la ciencia no son invenciones eminentemente jónico-griegas, de hecho, como Dussel (2009a), atinó a decir:

El mismo Aristóteles reconocerá, como en la República Platón (en la que éste describe idealmente una aldea egipcia, después de su viaje a Sais, la gran metrópoli de Atenas en el delta del Nilo), que las comunidades de sabios egipcios son el origen de la sabiduría de los mismos griegos: Aquellas ciencias que no van encaminadas ni a los placeres de la vida ni a atender sus necesidades, vieron entonces la luz primera y precisamente en aquellos lugares donde podía dedicarse al ocio (eskhólasan). Así ocurrió con las matemáticas en Egipto, porque en aquel país las comunidades de sabios (hiréon éthnos) estaban libres de todo trabajo manual (skholázein) (pág. 6).

Los egipcios a su vez fueron influenciados por los pueblos bantúes del sur del Sahara; y siglos antes de que se creara la academia de Platón ya habían existido otras escuelas de sabios como el calmecac en México y la edduba en Mesopotamia. En fin, hubo diversas expresiones precientíficas y prefilosóficas que se pierden en la noche de los tiempos y que, incluso, se 
pueden reconocer a comienzos de la hominización; al respecto, Lévi-Strauss (1984), concluyó: el hombre del neolítico o de la protohistoria resulta ser el heredero de una larga tradición científica (pág. 33).

De forma que otorgarle la invención de la duda sistemática a jonios y griegos es tan mítico como fijar el primer científico en la historia de la humanidad, pues, como advirtió Schneider (2005c), aunque:

El primer científico del mundo, se nos dice, fue Tales (640-546 a.C.). Esto es tan poco probable como probable es que podamos descubrir quién fue el primer científico en rigor de verdad. Pero esto carece de mayor sentido. Lo importante es que Tales fue el primer hombre del que sabemos e intuimos, a juzgar por lo que sabemos de él por terceros, que puede llamarse científico (pág. 11).

Asimismo, atribuirle el surgimiento de la ciencia al siglo XVII en Europa también es un dislate, pudiéndose mencionar varios casos científicamente meritorios en la restrictiva Edad Media y en otros puntos geográficos: Jean Buridán ya había creado la teoría del ímpetu para explicar el movimiento de proyectiles (concepto inercial clave con el que se anticipó a Newton); Nicolás Oresme descubrió el cambio de dirección de la luz a través de la refracción atmosférica (adelantándose a Robert Hooke); los matemáticos Richard Swineshead, William Heytesbury y John Dumbleton elaboraron el teorema de la velocidad media (le serviría a Galileo para desarrollar su Ley de la caída de los cuerpos); y sobre el particular Dussel (2009b), aportó que:

En el 820 d.C.: se publica un tratado de álgebra de Mohammet Ibn-Musa (que traducido en Europa en el siglo XVI significó todavía un adelanto sobre el tema en las matemáticas de ese tiempo). En óptica, astronomía, química, farmacia, medicina, el mundo musulmán estuvo unos 400 años adelantado a la periférica Europa (pág. 25).

Sin embargo, lo que sí puede reconocerse con Schneider (2005d), como hecho notable, es que: 
En Grecia [es] donde se produce un cuello de botella sin precedentes, donde se amontonan ideas y escuelas lideradas por aquellos que habían pensado en esas nuevas ideas, y surge un debate y contrapunto entre ideas disímiles, todo lo cual genera un efecto derrame positivo sobre otros hombres y escuelas y así sucesivamente, permitiendo una evolución explosiva del conocimiento (pág. 5).

Pudiendo entonces decirse que se trató del epicentro a partir del cual se desarrolló con mayor fuerza el fenómeno de la especialización que siglos más tarde mutaría en la hiperdisciplinariedad y la hiperespecialización, y el asignaturismo en el que el sistema educativo común ha venido cuadriculando estudiantes con mentalidad convergente; fenómenos radicales que hoy el pensamiento complejo busca reencauzar hasta su completa disolución para crear en su lugar puentes transdisciplinarios que, como advirtieron en el Convento de Arrábida (1994): "no excluye[n] la existencia de un horizonte transhistórico" (art. 6).

Siguiendo el curso de esta historia de agrietamientos ilustrativos y entrecruzamientos entre lo vulgar y lo selecto, se debe adicionar que la filosofía y la ciencia, que durante los últimos siglos se han venido reconociendo como áreas y campos de saberes distintos, era en tiempos antiguos un cuerpo indivisible, es decir, apuntó Russell (2013b): habían nacido juntas (pág. 32). Indivisibilidad que lentamente se fue difuminando hasta alcanzar un punto de inflexión notable a principios de la Edad Moderna sin que el resquebrajamiento se detuviera, llegando a obtener las comprometedoras fisuras que hoy continúan profundizándose. Desde luego, la filosofía Occidental (que deba decirse, muchas de sus antiguas escuelas fueron inicialmente movimientos políticos, morales y religiosos) tendría que recorrer un largo camino de especulaciones y experiencias para desprenderse de las fantásticas deducciones del Oriente Próximo.

Los filósofos de la Antigüedad, por ejemplo, en medio de sus profundos 
pensamientos le atribuyeron durante largo tiempo un gran poder cosmogónico a elementos como la tierra, el agua, el fuego y el aire por considerarlos decisivos para vivir. Por eso Morin (2003): apuntó con razón suficiente que "la filosofía griega empieza como una magia grandiosa. Esta magia se hace filosófica en el instante en que se hace laica, se intelectualiza, se ordena, y no sólo en símbolos sino en ideas" (pág. 237). Asimismo, las creencias religiosas han permeado muchas posturas memorables a lo largo de la historia de la ciencia; al respecto, Hawking y Mlodinow (2010a), dieron algunos ejemplos:

Anaximandro escribió que todas las cosas surgieron de una sustancia primordial y a ella retornarán, 'a menos que paguen pena y castigo por su inequidad'. Y según el filósofo jonio Heráclito (535-475 a.C.) el sol se comporta como lo hace porque de otro modo la diosa de la justicia lo expulsaría del cielo (pág. 29).

Párrafos adelante la dupla Hawking y Mlodinow (2010b), refieren que: "incluso tan tarde como el siglo XVI, el gran astrónomo alemán Johannes Kepler (1571-1630) creyó que los planetas tenían percepción sensorial y seguían conscientemente leyes de movimiento captadas por su 'mente'” (pág. 30).

Era entonces común a principios de la historia que la ciencia empírica se mezclara con reflexiones filosóficas, incluso con las especulaciones más supersticiosas. Eran épocas de la historia antigua en la que no existía diferencia entre las matemáticas y el estudio de la historia, la poesía o la política, era ni más ni menos la sabiduría misma, la totalidad del saber humano en su momento; solo que más adelante algunos individuos curiosos indagarían la realidad material desde las ciencias naturales (como Leucipo, Demócrito y Aristóteles); otros en la forma y el número en el marco de las ciencias exactas (Pitágoras y Euclides, por ejemplo); y algunos con la metafísica en el interior del sujeto y en la sociedad, el ser, el devenir (con Sócrates, Platón, Aristóteles, entre otros). 
Pero, ¿cómo y cuándo empezó a fracturarse este acervo sapiencial? Bueno, de nuevo debe decirse que precisar algo así es imposible puesto que se trató de un proceso complejo y no teleológico en el que las divisiones disciplinales se presentaron en diferentes grados, momentos y circunstancias. Sin embargo, una primera división o separación filosófica destacable que dio lugar a diferentes ramas fue la que llevó a cabo Aristóteles cuando clasificó una diversidad de objetos de estudio que hicieron brotar varias ciencias, lo que propició una rivalidad sobre cuál modo de conocer respondía mejor a la cuestión de la realidad, si las creencias razonables o la episteme, la interpretación o la explicación, lo sensible o lo inteligible, lo abstracto o lo determinado (todo ello en el periodo alejandrino: cerca del 400 a.E.C. al 100 d.E.C.) iniciándose así la llamada época de los especialistas.

Con respecto a la jerarquización de los saberes, Pitágoras jugó un papel decisivo al introducir las matemáticas (dianoia) como argumento deductivodemostrativo por excelencia; y Platón las consideró antesala de las ideas; las matemáticas ocuparían entonces un lugar preponderante en las demás Edades de la historia gracias al reconocimiento, hiperbolizado en la mayoría de casos, que harían de ellas muchos pensadores. Por ejemplo, como rescataron Hernández y Salgado (2010): Galileo aseguró que la naturaleza estaba escrita en el lenguaje de las matemáticas, Spinoza pretendió ordenar geométricamente la razón ética y Descartes consideró que el principio del conocimiento deja de ser el objeto y pasa a ser el sujeto, quedando los sentidos como criterios de conocimiento de clara inferioridad y desprestigio (pág. 7).

Así, a partir del siglo XVII las fracturas de la filosofía empiezan a agudizarse mucho más, a ramificarse en ciencias más específicas que buscaban su propia identidad y autonomía; especialmente las naturales, que venían haciendo investigación empírica desde la baja Edad Media. Ya en el Siglo XVIII era prácticamente imposible reconocer el conjunto de 
conocimientos que los griegos asumían como una unidad, como un tronco común (concepción monista de la ciencia). Posteriormente, hacia la segunda mitad del Siglo XIX se instauran "formalmente" las ciencias sociales, es en este siglo cuando, según Morin (2010a): la organización disciplinaria fue instituida, particularmente con la formación de las universidades modernas, y luego se desarrolló en el Siglo XX con el impulso de la investigación científica (pág. 1).

Entre tanto, los movimientos positivistas y materialistas, que no solo se limitaron a estudiar lo físico sino que optaron también por las consideraciones abstractas que venían promoviendo los círculos dialécticos e idealistas, se convirtieron en una tendencia científica que se fue sedimentando con mayor fuerza debido a que los objetos de estudio eran considerados como algo ajeno al sujeto, dando lugar al proceso de objetivización, es decir, incentivó la conquista por el objeto puro, por conocer de qué está hecha la realidad, cómo funciona el mundo y cuáles son sus leyes y estructuras; un fenómeno que contribuyó de manera importante a la parcelación disciplinaria, a los fraccionamientos teóricos y metodológicos, trayendo consigo sus propias formalizaciones y fronteras, distanciándose aún más de las especulaciones de la filosofía natural antigua. Para esta época empieza a robustecerse la hiperdisciplinariedad, haciéndose cada vez más rígida y custodiada por sus adeptos, quienes se apropiaban de "sus" saberes (y aún muchos lo hacen) como sus parcelas privadas, impidiendo la interacción con otras disciplinas, es decir, el edificio de la ciencia se había convertido en una 'propiedad horizontal' ocupada por una suerte de terratenientes del saber.

En consecuencia, las disciplinas se fueron distanciando entre ellas en su carrera por conocer el funcionamiento exacto y esencial de las cosas, y darle así alcance a la objetividad plena, a la realidad última. $Y$ es como el positivismo o materialismo lógico fue erigido como el modelo científico por excelencia, reconociéndose como sinónimo absoluto de ciencia, es decir, como el único método seguro para obtener conocimiento válido mediante la 
supuesta posesión de un conjunto de reglas objetivas y universales; modelo cientificista que aún continúa vigente y venerado en muchos escenarios académicos e investigativos hasta el punto de menospreciar, o sepultar, a su propia madre: la filosofía. Pongamos por cosa la declaración de Hawking y Mlodinow (2010c): quienes ante las preguntas ¿cómo podemos comprender el mundo en que nos hallamos? ¿Cómo se comporta el universo? ¿Cuál es la naturaleza de la realidad? ¿De dónde viene todo lo que nos rodea? ¿Necesitó el universo un Creador? Afirmaron que tradicionalmente esas son cuestiones para la filosofía, pero que la filosofía ha muerto, puesto que ella no se ha mantenido al corriente de los desarrollos modernos de la ciencia, en particular de la física (pág. 11).

En este aparte debe decirse que la ignorancia que evidenciaron estos destacados físicos teóricos sobre los aportes que le ha dispensado la filosofía a la ciencia (mediante noología, antropología biológica, antropoética, naturalismo epistemológico, filosofía de la mente, etc.), ha sido supina. Añádase a esta réplica la tesis de Bunge (2005a): que sostiene que en realidad la filosofía y la ciencia nunca se dividieron sino que se han venido traslapando parcialmente, puesto que siempre han compartido conceptos, principios y problemas claves muy generales, incluyendo conceptos universales como 'todo', 'y', 'algunos' 'son' y 'o' (incluyendo el signo de la coma) que la lógica los ha estudiado, así como 'acerca de' e 'inexacto' que pertenecen a la semántica (emparentada con la lógica), y que finalmente forma parte de la filosofía y de las matemáticas (pág. 13); rematando, Bunge (2005b), en esta defensa:

Los conceptos de materia, proceso, naturaleza, espacio, tiempo, sistema, historia, sociedad y artefacto son muy generales; no son propiedad exclusive de una ciencia particular y su análisis y sistematización están a cargo de la ontología (o metafísica), una de las ramas más antiguas de la filosofía. Los conceptos de conocimiento, ciencia, modelo y contrastabilidad pertenecen a la gnoseología (teoría del conocimiento), otra rama de la filosofía (pág. 14). 
Pero lo más curioso es que el mismo positivismo fue el que impulsó el desarrollo de las "ciencias sociales 'positivas"' en el Siglo XVIII con el propósito de estudiar la realidad sociohumana desde la experimentación para comprobar hipótesis y obtener conocimientos objetivos. Surgen entonces, con Augusto Comte en el Siglo XIX, desde la perspectiva mecanicista de las ciencias naturales y físicas: la economía, la antropología y la sociología como ciencias autónomas; luego aparecería la psicología y después la pedagogía... En síntesis, en palabras de Wallerstein (2006a), sucedió que:

En el curso del siglo XIX las diversas disciplinas se abrieron como un abanico para cubrir toda una gama de posiciones epistemológicas. En un extremo se hallaba primero la matemática (actividad no empírica), y a su lado las ciencias naturales experimentales (a su vez en una especie de orden descendente de determinismo, física, química, biología) (pág. 12).

¿Pero qué ocurrió en este episodio transicional con las humanidades y las ciencias sociales? Sobre el rumbo o posicionamiento de estos saberes en la academia, Wallerstein (2006b), relató:

En el otro extremo estaban las humanidades (o artes y letras), que empezaban por la filosofía (simétrica de la matemática como actividad no empírica) y junto a ella el estudio de prácticas artísticas formales (literatura, pintura, escultura, musicología) y llegaban a menudo en su práctica muy cerca de la historia, una historia de las artes. Y entre las humanidades y las ciencias naturales así definidas quedaba el estudio de las realidades sociales con la historia (idiográfica) más cerca de las facultades de artes y letras, y a menudo parte de ellas, y la "ciencia social" (nomotética) más cerca de las ciencias naturales (pág. 12).

No obstante, es válido agregar que se pueden identificar muchos brotes de ciencias sociales siglos antes del XIX, ya que durante los Siglos XVI y XVII estos saberes ya venían cobrando gran fuerza en Europa, especialmente en la economía con escritores importantes como Raymond de Quesnay y Adam 
Smith, y en la política con Maquiavelo, Juan Bodino y Thomas Hobbes, entre otros; e incluso mucho antes se pueden identificar tratados sociales como las obras de Platón como la República, Político, Las leyes y el Banquete (Siglo III a.E.C.); la Política de Aristóteles (Siglo IV a.E.C.) y el Código de Hammurabi (Siglo XVIII a.E.C.).

Ya en el siglo XX, paralelo al desmoronamiento del positivismo, iniciado por agudos filósofos de la ciencia como Kuhn, Popper, Lakatos y Feyerabend, surge la necesidad de desarrollar nuevos modelos y metodologías de investigación para abordar la realidad humana, puesto que se trataba de un fenómeno muy dinámico y contingente, con altos grados de incertidumbre, difícil de reglar y de predecir; en el que el investigador no está separado del objeto de estudio (objetividad) sino que forma parte de él (subjetividad), y en el que la misma ciencia es considerada un producto social e histórico; en consecuencia, aparecen nuevas propuestas científicas: la hermenéutica con Heidegger, Gadamer, Dilthey, Weber; la teoría crítica en la Escuela de Frankfurt con Adorno, Horkheimer, Habermas, Marcuse y Fromm; la etnometodología, la fenomenología y la teoría fundamentada con la escuela de Chicago; la etnografía de la mano de Boas, Malinowski, Hammersley, Atkinson, y demás personajes y ciencias que concurrieron e iniciaron la legitimación de sus saberes con diversas teorías, metodologías e instrumentos (la investigación acción participativa, las historias orales, el principio sistémico ecológico y las teorías de la acción comunicativa, de la investigación educativa, de la complejización de las ciencias, etc.) que hasta el día de hoy han hecho contribuciones esenciales en el esclarecimiento de las distintas realidades culturales, antropológicas, políticas, lingüísticas y demás dimensiones individuales, colectivas y contextuales.

Hasta aquí, las ciencias en mención se hallan en el marco de lo que Kuhn (2004b): calificó de "ciencia normal" (pág. 32); es decir, una estructura rígida, lineal y cerrada; a partir de la cual se empezó a gestar el pensamiento 
complejo y las ciencias de la complejidad como modos subversivos y sintetizadores de examinar la realidad y de ayudar a resolver problemas contraintuitivos y de frontera, constituyéndose así en una revolución filosóficocientífica sin parangón que ha venido tratando de trascender los propios campos y áreas de investigación junto con sus objetos de estudio para terminar concentrándose en la resolución de los problemas propiamente dichos, haciendo de la ciencia una estructura abierta y horizontal. Afortunada conmoción paradigmática de la cual surgieron enfoques sistémicos que inicialmente fueron abanderados por pensadores como Heinz von Foerster, Ludwig von Bertalanffy, Magoroh Maruyama, Jay Forrester, Gregory Bateson, Illya Prigogine, Isabelle Stenger, Humberto Maturana y Francisco Varela, e impulsados por pensadores como Edgar Morin, Niklas Luhmann, Mauro Ceruti, Basarab Nicolescu y Jean Louis Le Moigne, entre otros complejólogos. Lo que dio lugar a institutos de investigación en los que hoy confluyen diversos lenguajes, enfoques filosóficos, posturas científicas, metodologías y tradiciones.

No obstante, aún la propuesta organizacional de la clasificación de las ciencias continúa haciéndole creer a muchos expertos que la realidad está en verdad segmentada, y que por ende debe ser estrictamente abordada de forma sectorizada, uniprofesional y unidireccional; acuartelando así a las comunidades académicas y científicas en sus áreas, campos, programas y objetos de estudio; al punto que aún dentro de las mismas ciencias sociales y humanas también se presentan ópticas disciplinales deseslabonadas e independentistas. Sobre esta limitada posición, la de comprender la realidad de manera unidimensional, Morin (1988), manifestó:

La visión no compleja de las ciencias humanas, de las ciencias sociales, implica pensar que hay una realidad económica, por una parte, una realidad psicológica, por la otra, una realidad demográfica más allá, etc. Creemos que esas categorías creadas por las universidades son realidades, pero olvidamos 
que, en lo económico por ejemplo, están las necesidades y los deseos humanos (pág. 100).

\section{Conclusiones abiertas}

Si bien las actuales sociedades del conocimiento y de la información nos sitúan en una época privilegiada de la historia, aún muchos académicos e investigadores desconocen que en la historia de la razón científica las ideas, conceptos, concepciones, teorías, leyes y metodologías de los respectivos campos del conocimiento tienden entre ellos mismos a migrar, filtrarse y entremezclarse con gran fecundidad, proclives a la simbiosis, la metamorfosis y la interdependencia. No obstante, la mayoría de currículos oficiales siguen hoy anclados en apelotonamientos unidisciplinares $\mathrm{y}$, como lo advierte Maldonado (2015), aún continúan prevaleciendo congresos exclusivos sobre disciplinas en particular, ejemplo de ello: "los economistas se reúnen en congresos de economía hablan de ésta y publican en revistas de economía. Y así, sucesivamente, para los físicos, los matemáticos y demás" (pág. 46).

Por ello, para Morin (2010b), es importante reconocer que:

La historia de la ciencias no es solamente la de la constitución y de la proliferación de las disciplinas sino también aquella de la ruptura de las fronteras disciplinarias, de la usurpación de un problema de una disciplina sobre otra, de circulación de conceptos, de formación de disciplinas híbridas que van a terminar por atomizarse, en fin, es también la historia de la formación de complejas y diferentes disciplinas que van a ir sumándose a un tiempo que aglutinándose, dicho de otro modo, si la historia oficial de la ciencia es la de la disciplinariedad, otra historia ligada e inseparable, es la de la Inter-trans-poli-disciplinariedad (pág. 3).

De allí la necesidad de que los pensadores, tanto los convergentes como los divergentes, eleven su nivel de conciencia emocional y transdisciplinaria, de tal forma que puedan entregarse con mente abierta a dinámicas académico investigativas solidarias y autocríticas, con la capacidad 
de compartir, replantear y crear métodos, conceptos y lenguajes que les permitan redescubrirse y profundizar aún más en los diversos matices, interfaces y niveles multirreferenciales de la realidad. Y en esto, el alma máter, como apunta Agüero (2019), puede aportar: "abriendo espacios para la emergencia de posturas heurísticas y creativas que intenten modelar pensamientos, sin pretender convertirse en nuevos dogmas, sino que constituyan otra manera de repensar en escenarios complejos y múltiples, no de forma aislada, unívoca, reductiva" (pág. 160). Total, la filosofía de la ciencia, la historia de la ciencia y el pensamiento complejo son fuentes de conocimiento de las que las comunidades educativas e investigativas deberían surtirse siempre para elevar aún más su alfabetización científica y maximizar sus procesos creativos.

\section{Referencias}

Agüero, M. (2019). La Transcomplejidad como Agente Integrador de las Tecnologías de Información y Comunicación. Revista Scientific, 4(11), 156-176, e-ISSN: 2542-2987. Recuperado de:

https://doi.org/10.29394/Scientific.issn.2542-2987.2019.4.11.8.156-176

Alonso, C. (2004). La agonía del cientificismo. Una aproximación a la filosofía de la ciencia. Navarra, España: Eunsa Ediciones.

Bunge, M. (1983). La ciencia: su método y su filosofía. Buenos Aires, Argentina: Siglo XXI Editores.

Bunge, M. (2005a,b). Buscar la filosofía en las ciencias sociales. $2^{\text {da }}$ Edición. México: Siglo XXI Editores.

Cavada, D. (2004). Las ciencias naturales y las ciencias sociales: un debate sobre su acercamiento interparadigmático. Tesis para optar al título de Sociólogo. Chile: Universidad de Chile. Recuperado de: http://repositorio.uchile.cl/handle/2250/106411

Convento de Arrábida (1994). Carta de la Transdisciplinariedad. Setúbal, 
Portugal: Primer Congreso Mundial de Transdisciplinariedad. Recuperado de:

http://redcicue.com/attachments/article/137/2.0\%20CARTA\%20DE\%2 OLA\%20TRANSDISCIPLINARIEDAD.pdf

Delgado, C. (2011). Hacia un nuevo saber. La bioética en la revolución contemporánea del saber. La Habana, Cuba: Editorial Acuario.

Dussel, E. (2006). Filosofía de la liberación. $4^{\text {ta }}$ Edición. Bogotá, Colombia: Editorial Nueva América.

Dussel, E. (2009a,b). Ética de la liberación: historia mundial de las eticidades. Madrid, España: Editorial Trotta, 661 págs.

García, E. (2008). Aproximación epistemológica al concepto de ciencia: una propuesta básica a partir de Kuhn, Popper, Lakatos y Feyerabend. Andamios. Revista de Investigación Social, 4(8), 185-212, e-ISSN: 1870-0063. Recuperado de:

http://www.redalyc.org/articulo.oa?id=62811458008

Hawking, S., \& Mlodinow, L. (2010a,b,c). El gran diseño. Barcelona, España: Editorial Crítica.

Hernández, F., \& Salgado, S. (2010). El racionalismo de Descartes: La preocupación por el método. DUERERÍAS - Cuadernos de Filosofía, 1-19, e-ISSN: 1989-7774. Recuperado de:

http://guindo.pntic.mec.es/ ssag0007/filosofica/Descartes.pdf

Hernández, L. (2014). La formación integral desde lo curricular en los ingenieros agrónomos en la FUM Manatí. Revista semestral de investigación de la Corporación Internacional para el Desarrollo Educativo - CIDE, 1(14), 168-174, e-ISSN: 1909-955X. Recuperado de: http://cide.edu.co/investigacion/images/revista14/15.SI.\%20La\%20for maci\%C3\%B3n\%20integral\%20desde\%20lo\%20curricular\%20fun\%20 manati.pdf

Kuhn, T. (2004a,b). La Estructura de las Revoluciones Científicas. $8^{\text {va }}$ 
Reimpresión. México: Fondo de Cultura Económica, 318 págs.

Lévi-Strauss, C. (1984). El pensamiento salvaje. México: Fondo de Cultura Económica.

Maldonado, C. (2015). Introducción al pensamiento científico de punta, hoy. Bogotá, Colombia: Ediciones Desde Abajo.

Morin, E. (2003). El hombre y la muerte. $4^{\text {ta }}$ Edición. Barcelona, España: Editorial Kairós.

Morin, E. (1988). El Paradigma de Complejidad. De Culture, signes, critiques. Colección Symbolique et idéologie, nro. S 16. Canadá, Estados Unidos: Presses de L'Université de Québec. Recuperado de:

http://www.pensamientocomplejo.org/docs/files/MorinEdgar Introducci on-al-pensamiento-complejo Parte2.pdf

Morin, E. (2010a,b). Sobre la interdisciplinariedad. Boletín No. 2. París, Francia: Centre International de Recherches et Etudes Transdisciplinaires (CIRET). Recuperado de:

http://www.pensamientocomplejo.org/docs/files/morin sobre la interdi sciplinaridad.pdf

Real Academia Española (2014). Diccionario Real Academia Española. Edición 23a. Madrid, España: Editorial Espasa.

Russell, B. (2013a,b). Historia de la Filosofía Occidental. Tomo I. Madrid, España: Grupo Editorial Planeta.

Schneider, J. (2005a,b,c,d). El Nacimiento de la Ciencia en los Presocráticos. Buenos Aires, Argentina: Universidad del CEMA. Recuperado de:

https://ucema.edu.ar/publicaciones/download/documentos/304.pdf

Wallerstein, I. (2006a,b). Abrir las ciencias sociales. $9^{\text {na }}$ Edición, Comisión Gulbenkian para la reestructuración de las ciencias sociales. México: Siglo XXI Editores. 


\section{Héctor de León Bedoya Leguizamón}

e-mail: hbedoya@unillanos.edu.co

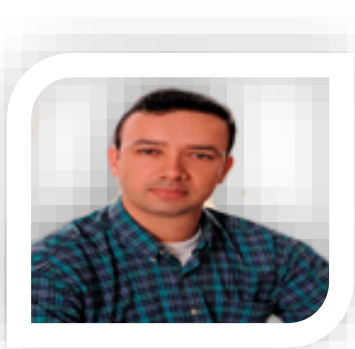

Oriundo de Villavicencio, Meta, Colombia, nacido el 17 de septiembre del año 1973. Licenciado en Educación física y deportes de la Universidad de los Llanos (UNILLANOS); Magister en educación de la Universidad Santo Tomás (UST); Doctorando en Pensamiento complejo en la Multiversidad Mundo Real; Docente de la Universidad de los Llanos; Publicaciones: Los vaivenes del clima institucional (2011), en la Revista Ímpetus; Revista Kinesis; La subjetividad, un arroyo insalvable (2012), en la Revista Educación física y deporte de la Universidad de Antioquia (UdeA); Carranza (Editorial Entreletras, 2013); Cuerpos sin alma; Cuerpos sin Dios (2018), editorial Unillanos; De excursión por la geografía de la libertad (2018), editorial Unillanos; entre otras.

El contenido de este manuscrito se difunde bajo una Licencia de Creative Commons ReconocimientoNoComercial-Compartirlgual 4.0 Internacional 